\title{
FOOD PROTEIN-INDUCED ENTEROCOLITIS SYNDROME IN PRETERM NEWBORNS
}

\author{
Enza D'Auria ${ }^{1}$, Ilaria Cocchi ${ }^{1}$, Giovanna Monti ${ }^{2}$, Marco Sartorio ${ }^{3}$, Irene Daniele ${ }^{4}$, Gianluca \\ Lista $^{4}$, and Gian Vincenzo Zuccotti ${ }^{1}$ \\ ${ }^{1}$ University of Milan \\ ${ }^{2}$ Ospedale Infantile Regina Margherita \\ ${ }^{3}$ V. Buzzi Children's Hospital, University of Milan \\ ${ }^{4}$ Neonatal Pathology and Neonatal Intensive Care Unit, "V. Buzzi" Children's Hospital, \\ University of Milano, 20154 Milano, Italy.
}

May 26, 2021

\section{FOOD PROTEIN-INDUCED ENTEROCOLITIS SYNDROME IN PRETERM NEWBORNS}

Enza D'Auria ${ }^{1}$, Ilaria Cocchi ${ }^{2}$, Giovanna Monti ${ }^{3}$, Marco Ugo Andrea Sartorio ${ }^{4}$, Irene Daniele $^{2}$, Gianluca Lista ${ }^{2}$, Gian Vincenzo Zuccotti ${ }^{4}$

${ }^{1}$ Allergy Unit-Vittore Buzzi Children's Hospital, University of Milan, Milan, Italy

${ }^{2}$ Neonatal Pathology and Neonatal Intensive Care Unit, Vittore Buzzi Children's Hospital, University of Milan, Milan, Italy.

${ }^{3}$ Department of Pediatric Allergology, Regina Margherita Children's Hospital, Turin, Italy.

${ }^{4}$ Department of Pediatrics, Vittore Buzzi Children's Hospital, University of Milan, Milan, Italy.

Correspondence: Enza D'Auria, Head Allergy Unit, Vittore Buzzi Children's Hospital, University of Milan, Milan, Italy.

Email: enza.dauria@unimi.it

CONFLICT OF INTEREST : The authors declare that there are no conflicts of interest.

FINANCIAL SUPPORT: None

KEYWORDS: FPIES, preterm newborns, twins, low birth weight, atypical

Key Messages statement: Food-protein-induced enterocolitis syndrome (FPIES) in preterm newborns may mimic necrotizing enterocolitis. A combination of family history and laboratory findings, e.g. eosinophilia plus leukocytosis with neutrophilia and thrombocytosis, could help to discriminate between these two conditions.

A dietetic approach with an elemental diet based on amino acid formula may be adopted as the first-line treatment when FPIES is suspected in preterm newborns.

To the Editor,

Food-protein-induced enterocolitis syndrome (FPIES) was not recognized as a specific disorder until the 1970s. While the European Academy of Allergy and Clinical Immunology (EAACI) Guidelines addressed FPIES in 2014,it was only in 2017 that the first international consensus guidelines for its diagnosis and 
treatment were published. The clinical presentation of FPIES can mimic countless different conditions, making its management challenging. In the neonatal period, especially in preterm babies, FPIES should be carefully differentiated from necrotizing enterocolitis (NEC), as its management, morbidity and mortality are remarkably different. To date, only a few anecdotal case reports of FPIES in preterm newborns and a case series from a US Neonatal Unit have been published .

We report six cases of preterm, low-body-weight newborns born between January 2014 and October 2020 at the Neonatal Intensive Care Unit (NICU) of Vittore Buzzi Children's Hospital in Milan or the Child Illness and Care Department, Regina Margherita Children's Hospital in Turin.

Data on pregnancy and birth, family history of atopy, onset symptoms and clinical course, laboratory and imaging findings, type of diet and timing of symptom resolution are summarized in Table 1.

Two of the six cases had a positive family history for allergic diseases. Four babies were delivered by caesarean section (CS) and two by vaginal delivery. Four cases were twins (of which one set was monochorionic); in each set, only one sibling was affected. Three of the cases were in males and three in females. The median gestational age (GA) was $33^{+3}$ (range $28^{+2}-36^{+6}$ weeks). All babies had a low birth weight, with a median birth weight of $1902 \mathrm{~g}$ (range $1070 \mathrm{~g}-1980 \mathrm{~g}$ ), and two (C1 and C2) were small for gestational age. The median age of onset was 12 days of life (median $\mathrm{GA} 34^{+6}$, min $30^{+5}$ ). In $3 / 6$ cases, the first symptoms appeared in the first week of life, and in one patient $(\mathrm{C} 1)$ in the first hours after delivery. All cases initially presented with vomiting (C1 bilious vomiting) and/or pathological stools (4/6 bloody stools); 4/6 had a distended and painful abdomen; 2/6 had unstable vital signs and a poor general condition. The median serial WBC was $14920 / \mathrm{mm}^{3}$ (range $6840-38780 / \mathrm{mm}^{3}$ ); neutrophilia was also observed, with a median count of $7371 / \mathrm{mm}^{3}$ (range $1370-24025 / \mathrm{mm}^{3}$ ). In $5 / 6$ cases, concomitant eosinophilia was observed (range $350-$ $\left.3102 / \mathrm{mm}^{3} ; 1.5 \%-11.4 \%\right)$. An increase in C-reactive protein was observed in $4 / 6$ cases. Culture samples (blood, urine and stools) were negative in all cases. The median platelet count was $357500 / \mathrm{mm}^{3}$ (range $\left.250000-650000 / \mathrm{mm}^{3}\right)$. In $3 / 6$ cases, anemia was also observed. Only C1 had serum-specific IgE antibodies (to beta-lactoglobulin and casein). Radiological findings were remarkable in $4 / 6$ cases, mostly showing bowel loop distension. Abdominal ultrasound showed intestinal pneumatosis in one case and distended bowel loops in $2 / 6$ cases.

Relapse on the reintroduction of the trigger food was observed in $5 / 6$ cases, without vomiting in two of these. The mean time from symptom onset to beginning the definitive diet was 23 days (range $5-60$ ). An oral food challenge was performed in $3 / 6$ cases and was positive in all three, with profuse vomiting.

The diagnosis and management of FPIES has recently been addressed in guidelines. Nevertheless, FPIES in preterm babies is still poorly understood, and is often associated with misdiagnosis or delayed diagnosis. Both FPIES and NEC often develop in infants fed with formula. Although the exact pathogenetic mechanism is still unknown, cow's milk has been hypothesized to act as a trigger of intestinal inflammation, activating the immune innate system and enhancing the lymphocyte response to cow's milk allergens. Many cases of NEC do not have a fulminant course, and can be managed conservatively. It is therefore important to differentiate between these conditions, as their management is very different. We observed eosinophilia in $5 / 6$ cases (three mild and one severe at onset, three moderate at relapsing episodes) during the clinical course of FPIES, in agreement with literature data. This finding, which is even more frequent in premature babies and those with low GA and low birth weight, may be an important indicator of FPIES, especially if combined with other laboratory findings, e.g. leukocytosis with neutrophilia and thrombocytosis. In contrast, thrombocytopenia and neutropenia are mostly observed in classic NEC.

Case $\mathrm{C} 1$, the monochorionic twin, is of particular interest, as his sibling was not affected and he showed serum-specific IgE antibodies to cow's milk proteins. To our knowledge, this is the first report of FPIES in a single affected monochorionic twin. A previous report described monochorionic twins who both developed FPIES with positive serum-specific IgE antibodies. Both our case $\mathrm{C} 1$ and the twins in the previous report presented early onset, marked eosinophilia and the presence of hematochezia.

It is well known that allergen sensitization can occur in utero. This scenario has been described for IgE- 
mediated food allergy, but recent reports point to the same background for FPIES .

Pneumatosis is recognized as a characteristic radiological feature of NEC, although its presence is not constant. Pneumatosis was observed in one of our cases (17\%), and in all 5 cases (100\%) in the US series (5). In both our series and the US series, imaging also revealed bowel loop distension, as is also seen in classic NEC. Imaging does not therefore seem to help differentiate between NEC and FPIES. Nevertheless, given the significant morbidity and mortality associated with NEC, evaluation by imaging is necessary in clinical practice.

In summary, a combination of different features, including a family history of atopy and laboratory findings such as eosinophilia, especially in association with leukocytosis, neutrophilia and thrombocytosis, could help to raise the suspicion of FPIES, although the diagnosis primarily remains a clinical one.

Following a diagnosis of FPIES, a dietetic approach should be adopted. It is worth noting that most of our patients failed to recover with an extensively hydrolyzed formula, while all responded to amino acid formula. This could have been due to their immature gastrointestinal gut, as immature absorption function is common in preterm babies (5). In premature infants, it may therefore be reasonable to start feeding with an elemental diet based on an amino acid formula.

In our opinion, an OFC may be indicated or considered to confirm the diagnosis in the event of a relapse-free course or a relapse without vomiting. The timing of any OFC is at the clinician's discretion, taking into account the patient's clinical condition and weight.

In conclusion, the diagnosis of FPIES in the neonatal period, even more in preterm newborns, is challenging. Increasing clinicians' awareness of FPIES in this specific population may help to avoid unnecessary antibiotic therapies, protracted parenteral nutrition and a typical relapsing course on reintroduction of the trigger food.

Further data are needed to differentiate FPIES from NEC more clearly and to improve its diagnosis and management.

\section{Hosted file}

TABLE 1..docx available at https://authorea.com/users/415934/articles/523693-food-proteininduced-enterocolitis-syndrome-in-preterm-newborns 